Revue

Revue de l'histoire des religions

de Ihistoire des religions

Annie Vigourt, Xavier Loriot, Agnès BerengerBadel et Bernard Klein (dir.), Pouvoir et religion dans le monde romain, en hommage à Jean-Pierre Martin

\title{
Arnaud Suspene
}

\section{OpenEdition}

Journals

Édition électronique

URL : http://journals.openedition.org/rhr/6683

DOI : $10.4000 /$ rhr.6683

ISSN : 2105-2573

Éditeur

Armand Colin

Édition imprimée

Date de publication : 1 septembre 2008

Pagination : 417-419

ISBN : 978-2200-92445-4

ISSN : 0035-1423

\section{Référence électronique}

Arnaud Suspene, «Annie Vigourt, Xavier Loriot, Agnès Berenger-Badel et Bernard Klein (dir.), Pouvoir et religion dans le monde romain, en hommage à Jean-Pierre Martin », Revue de l'histoire des religions [En ligne], 3 | 2008, mis en ligne le 14 janvier 2010, consulté le 10 décembre 2020. URL : http:// journals.openedition.org/rhr/6683 ; DOI : https://doi.org/10.4000/rhr.6683 
la narration. Ils sont une suite de prescriptions spatio-temporelles adressées au défunt, peut-être récitées par un officiant lors de la cérémonie funéraire ce qui expliquerait le jeu des énoncés en je et en tu à l'intérieur du texte.

De la lecture de l'un ou l'autre de ces étranges documents, l'auteur tire une structure spatio-temporelle ternaire qui mène du passé (vie mortelle) au futur (divinisation du défunt) en passant par le présent (plongeon dans l'Hadès). Comme dans les chapitres précédents, il propose une démarche comparative avec cette fois les représentations des Enfers dans l'iconographie apulienne, dans l'optique d'y souligner une fois encore les différences : les figures d'Orphée et de Dionysos dans la céramique apulienne offrent une représentation des Enfers différente de l'itinéraire décrit dans les lamelles d'or. Rappelant qu'il a voulu illustrer l'impasse à laquelle conduirait une étude des concepts spatio-temporels des Grecs qui ne serait menée qu'à la lumière de nos propres repères, Claude Calame conclut en préconisant dans le domaine des sciences humaines en général « une position de modestie relativiste » (p. 298). C'est en effet la leçon qu'il convient de tirer de cet ouvrage dense dont l'écriture complexe occulte parfois un contenu plus simple qu'il n'y paraît. L'auteur y propose une approche résolument novatrice, prenant ses distances par rapport aux méthodes généralement mises en œuvre dans l'analyse des textes anciens, même si un comparatisme centré sur les différences et les contrastes ne me semble pas moins relever « du résultat de la construction formalisante de l'anthropologue ou de l'historien » (p. 81) que le comparatisme traditionnel.

Carine VAN LIEFFERINGE, Université Libre de Bruxelles.

Annie Vigourt, Xavier Loriot, Agnès Berenger-BAdel et Bernard KLeIN (dir.), Pouvoir et religion dans le monde romain, en hommage à JeanPierre Martin, Paris, Presses de l'Université de Paris-Sorbonne, 2006, 606 p.-XVI p. de pl., 24 cm (« Passé-présent »), 40 €.

Ce gros volume contient les textes de 36 communications prononcées à la Sorbonne en l'honneur de Jean-Pierre Martin. Le livre s'organise en cinq grandes parties (Partie I : «De la République à l'Empire »; Partie II : «L'empereur : homme, prince et dieu »; Partie III : «Rituels et espaces »; Partie IV : «Elites sociales et religion »; Partie V : «Approches régionales : Occident et Orient »). L'ouvrage comprend en outre une introduction générale des éditeurs, une bibliographie de Jean-Pierre Martin, une bibliographie générale, un cahier couleur de 16 pages, une tabula gratulatoria et des conclusions par Jean-Pierre Callu.

Il est très difficile de rendre compte de manière générale d'un ouvrage aussi riche et qui réunit tant d'articles consacrés à des sujets si divers. Disons sans attendre que le résultat final a de quoi satisfaire le destinataire 
du volume : des documents nouveaux trouvent ici leur première publication ; des interprétations nouvelles et originales de sources déjà connues sont proposées avec audace et rigueur; des bilans sur des questions épineuses de l'historiographie romaine sont mis à la disposition de la communauté des historiens. C'est donc là un livre important qui rendra de considérables services à tous ceux qui s'y référeront.

Plus que le thème du colloque lui-même, ce sont les centres d'intérêt de Jean-Pierre Martin qui ont inspiré aux participants le sujet de leur communication. Comme ceux-ci sont nombreux et variés, le lecteur se voit offrir une très large gamme d'articles : les thèmes religieux sont naturellement très présents et l'on est ainsi introduit à la religiosité d'Hannibal, à l'appropriation par Rome et par le Prince des grands lieux de culte d'Italie, au rôle du christianisme dans la « crise » du troisième siècle, à l'adhésion réelle ou supposée des Romains à la divinité des empereurs, à l'emprise dionysiaque sur telle partie de la Domus aurea, aux représentations iconographiques de la piété à Pompéi, aux conséquences de l'implantation des lieux de culte sur la sociabilité religieuse, à l'histoire et aux fonctions du personnel affecté aux temples, à la gestion des conflits liés aux terres sacrées par le pouvoir romain, au polythéisme anatolien sous l'Empire dans ses rapports avec le pouvoir royal, au rôle des prêtrises et des inscriptions à caractère religieux dans la définition de la notabilité dans les Trois Gaules (deux articles très complémentaires), à l'importance de l'épigraphie religieuse pour la connaissance de la Germanie et de la Cisalpine, aux cultes militaires de Mithra et de Jupiter Dolichenus, à l'évolution du pouvoir sacerdotal en Judée à l'époque romaine et aux missions très administratives de l'Archiereus d'Alexandrie et d'Egypte.

La plupart de ces communications font naturellement le lien, dans la mesure du possible, entre religion et pouvoir. Certains intervenants sont allés plus loin en consacrant spécifiquement leur enquête à la légitimation du politique par le religieux : ainsi sont étudiés les nouvelles fonctions du Puluinar du Grand Cirque à l'époque impériale (communication qu'on complétera par l'article de Jean-Louis Ferrary dans Archiv für Religionsgeschichte 1.2, 1999, p. 215-232), le rapport entre les généalogies divines et les prétentions politiques de la noblesse républicaine, et trois aspects du culte impérial (les Flamines de la contributio cirtéenne, la dévotion au prince de l'armée d'Afrique et l'allure particulière du culte impérial dans le Pont). Certaines communications sont plus spécialement consacrées à la question du pouvoir : le lecteur se voit offrir une synthèse détaillée sur l'âge du pouvoir à Rome sous la République, une étude captivante des conditions spatiales de l'activité des magistrats, des tribuns et de certains prêtres, un aperçu sur le régime de vie de Sévère Alexandre, où la référence médicale paraît essentielle, et une brève présentation des ueteres principes tels qu'ils apparaissent chez Ammien Marcellin. Enfin, et c'est là un hommage à l'éclectisme de Jean-Pierre Martin, ce volume comprend plusieurs articles utilisant plus spécialement les diverses disciplines historiques fondamentales 
pour l'étude de l'Antiquité. On a déjà mentionné la peinture pompéienne et le décor de la Domus aurea, mais l'archéologie et la sculpture ne sont pas non plus négligées : les nouvelles découvertes sur le chef-lieu de la cité des Leuques viennent enrichir un dossier utilement présenté, une statue de Vaison-la-Romaine fait l'objet d'une nouvelle description et un bloc de calcaire retrouvé à Reims et représentant une femme donnant le sein complète la documentation iconographique de la Reims antique.

L'épigraphie est abondamment représentée et parfois de manière spectaculaire : un nouveau poids de plomb jette un nouvel éclairage sur le dossier de L. Egnatius Victor Lollianus. Alliée aux sources littéraires, l'épigraphie permet aussi de connaître les dévotions privées des gouverneurs de province et de distinguer la nature d'Aiôn dans deux documents pourtant contemporains. L'intérêt de comparaisons entre les diverses sources ressort clairement de l'étude minutieuse des représentations antiques du lièvre ; complétée d'une analyse anthropologique des sacrifices sanglants dans le monde celte, cette étude conduit à minimiser l'importance de la rhétorique dans la description des massacres perpétrés par Boudicca.

Les familiers de l'œuvre de Jean-Pierre Martin ne seront pas surpris de trouver aussi dans ce volume deux communications consacrées à la numismatique : un brillant article de synthèse et de méthode consacré aux rapports de la politique et de la religion dans le monnayage de la fin de la République (qu'on pourra enrichir des réflexions sur la libération typologique du denier présentées par Jonathan Williams et Andrew Meadows in Journal of Roman Studies 91, 2001, 27-49) et une analyse du type du laboureur (qui aurait sans doute gagné à s'appuyer sur les documents de travail les plus récents et non sur le vieux catalogue de Grueber). Ce volume correspond donc admirablement à son dédicataire : on y traite avec pénétration de la religion et du pouvoir, en exploitant avec bonheur toutes les branches de l'histoire ancienne.

Arnaud SUSPENE, Université d'Orléans/IRAMAT-Centre Ernest-Babelon.

Andreas MERKT, Das Fegefeuer. Enstehung und Funktion einer Idee, Darmstadt, Wissenschaftliche Buchgesellschaft, 2005, 21,5 cm, 131 p., $29,90 €$ €.

A. Merkt donne le ton dès l'introduction et expose buts et motivations avec une clarté réjouissante. Ainsi, le nom de Jacques Le Goff apparaît à la première ligne, en-tête programmatique. Un historien ne peut, même un quart de siècle après sa parution, aborder la question du purgatoire sans se prononcer sur La Naissance du Purgatoire (Paris, 1981). Il peut en revanche s'appuyer sur l'autorité de J. Le Goff pour ensuite se positionner et organiser sa matière. Selon A. Merkt, J. Le Goff raisonne en médiéviste. 